\section{GW23-e2341 RETROGRADE CORONARY VENOUS DELIVERY OF FIBROBLAST GROWTH FACTOR-2 ENHANCES IN VIVO HOMING AND DIFFERENTIATION OF MESENCHYMAL STEM CELLS}

doi:10.1136/heartjnl-2012-302920b.9

Shaoping Nie, Xiao Wang, Shibin Qiao, Lei Zhen, Jianzeng Dong, Xiaohui Liu,

Changsheng Ma, Shaoping Nie. Beijing Anzhen Hospital, Capital Medical University

Objectives In vitro studies showed fibroblast growth factor-2 (FGF-2) controls migration of mesenchymal stem cells (MSCs). In the present study, we established concentration gradient of FGF-2 between coronary venous blood and target myocardium by coronary venous retroperfusion and assessed the effects of FGF-2 gradient on in vivo homing and differentiation of MSCs. Methods Acute myocardial infarction (AMI) was induced by ligation of left anterior descending (LAD) coronary artery. First, twelve animals were randomised into 4 groups based on the time of balloon dilation (retroperfusion time), being $0 \mathrm{~min}$ $(n=3), 5 \mathrm{~min}(\mathrm{n}=3), 10 \mathrm{~min}(\mathrm{n}=3), 15 \mathrm{~min}(\mathrm{n}=3)$. One week after AMI, FGF-2 was retrogradely perfused into anterior interventricular vein in parallel to $\mathrm{LAD}$, and FGF-2 concentrations of serum and myocardial tissue were measured by ELISA. The time of FGF-2 gradients after coronary venous retroperfusion was evaluated. Second, ten animals were randomised to MSCs group $(n=5)$ and FGF-2+MSCs group $(n=5)$ after AMI. MSCs were cultured and transfected by pLEGFP-N1 vector before transplantation. One week after retroperfusion, EGFP-positive endothelial cells and cardiomyocytes in infarcted myocardium were compared between the two groups by immunofluorescence method.
Results First, there were statistical differences regarding FGF-2 concentration between coronary venous blood and infarcted myocardium at $5 \mathrm{~min}(81.13 \pm 15.07 \mathrm{pg} / \mathrm{ml}$ vs $210.66 \pm 65.09 \mathrm{pg} /$ $\mathrm{ml}, \mathrm{p}<0.05)$ and $10 \mathrm{~min} \quad(59.80 \pm 12.15$ vs $124.92 \pm 21.31$, $\mathrm{p}<0.05)$. No difference was found between the two tissues at 15 min. Remarkable FGF-2 gradient can be established between coronary venous blood and infarcted myocardium at 5-10 min after retroperfusion. Second, FGF-2+MSCs group showed more EGFP-positive cells compared with MSCs group. The number of endothelial cells $\left(23.8 \pm 6.2 / \mathrm{mm}^{2}\right.$ vs $\left.11.4 \pm 2.9 / \mathrm{mm}^{2}, \mathrm{p}<0.05\right)$ and cardiomyocytes $\left(11.3 \pm 2.5 / \mathrm{mm}^{2}\right.$ vs $\left.8.3 \pm 2.2 / \mathrm{mm}^{2}, \mathrm{p}<0.05\right)$ originating from the injected cells was more in the FGF-2 +MSCs group than in the MSCs group.

Conclusions A stable FGF-2 concentration gradient can be established in vivo between coronary venous blood and infarcted myocardium at 5-10 min after retroperfusion, which can promote homing of MSCs into the infarcted myocardium and differentiation. 